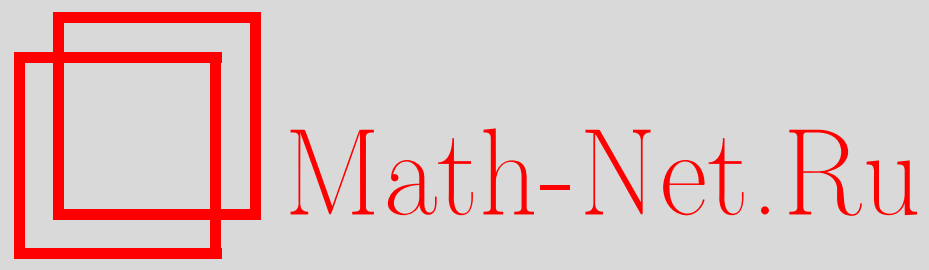

А. Г. Асланян, Собственные значения оператора теории оболочек в зоне регулярного вырождения, Функи. анализ и его прил., 1998, том 32, выпуск 4, 72-74

DOI: https://doi.org/10.4213/faa439

Использование Общероссийского математического портала MathNet.Ru подразумевает, что вы прочитали и согласны с пользовательским соглашением http://www . mathnet.ru/rus/agreement

Параметры загрузки:

IP : 3.85 .183 .62

26 апреля 2023 г., 14:30:18

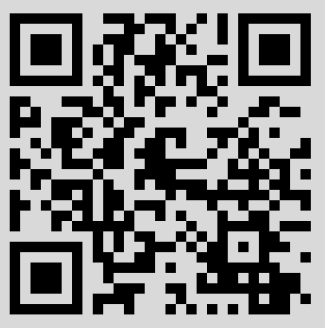




\title{
ЛИТЕРАТУРА
}

1. Kottman C. A. Trans. Am. Math. Soc., 150, 565-576 (1970). 2. Rankin R. A. Proc. M. A. Glasgow, 2, 139-144 (1955). 3. Burlak J. A. C., Rankin R. A., Robertson A. P. Proc. M. A. Glasgow, 4, 22-25 (1958). 4. Wells J. H., Williams L. R. Imbeddings and extensions in analysis, Springer-Verlag, Berlin, 1975. 5. Hudzik $H$. Collect. Math., 44, 131-135 (1993). 6. Clever C. E. Pacific J. Math., 65, 325-335 (1976). 7. Rao M. M., Ren Z. D. Theory of Orlicz spaces, M. Dekker, N.Y., 1991. 8. Hudzik H., Landes T. Bull. U.M.I., (7) 9-A, 225-237 (1995). 9. Rao M. M., Ren Z. D. Studia Math., 126, No. 3, 235-251 (1997). 10. Ball K. Israel J. Math., 59, 243-256 (1987). 11. Elton J., Odell E. Colloq. Math, 44, 105-109 (1981). 12. Lindenstrauss J., Tzafriri L. Classical Banach spaces. Function spaces. II, Springer-Verlag, Berlin, 1979. 13. Крейн С. Г., Петунин Ю. И., Семенов Е. М. Интерполяция линейных операторов, Наука, M., 1978. 14. Pisier G. J. d'Analyse Math., 35, 264-281 (1979). 15. Franchetti C., Semenov E. M. C. R. Acad. Sci. Paris, 323, 723-728 (1996). 16. Carothers N. L., Dilworth S. J. Texas Func. Anal. Sem., 1985-1986, pp. 107-133. 17. Figiel T., Johnson W. B., Tzafriri L. J. Approx. Theory, 13, 395-412 (1975).

Университет Вюрцбурга

Воронежский государственный университет
Поступило в редакцию 10 февраля 1998 г.

УДК 517.9

\section{Собственные значения оператора теории оболочек в зоне регулярного вырождения}

\author{
(C) 1998. А. Г. АСЛАНЯН
}

Рассматриваются свободные колебания тонкой упругой оболочки произвольного очертания. Срединная поверхность оболочки $S$ предполагается выпуклой и достаточно гладкой, ее граница $\gamma$ - кусочно гладкой. На поверхности $S$ вводится ортогональная система координат $\alpha, \beta$, отнесенная к линиям кривизны $((\alpha, \beta) \in S)$. Указанные колебания описываются системой трех дифференциальных уравнений в перемещениях [1]

$$
\left(\mu^{4} N+L\right) u=\lambda u \text {. }
$$

Здесь $N$ и $L$ - матричные дифференциальные операторы; их явный вид ввиду громоздкости мы не приводим (см., например, [1, с. 8; 40, 41]). Заметим, что вид оператора $N$ зависит от выбора соотношений упругости в теории оболочек [2, с. 11]. Однако сформулированный ниже результат справедлив во всех наиболее часто встречающихся вариантах соотношений упругости (см. $[3$, c. $299 ; 4$, c. $55-58])$.

Далее, $u(\alpha, \beta)=\left(u_{1}, u_{2}, u_{3}\right)$ - вектор перемещения точки срединной поверхности оболочки (компонента $u_{3}$ направлена по нормали к поверхности $S$, а компоненты $u_{1}, u_{2}$ лежат в касательной плоскости), $\lambda$ - спектральный параметр, пропорциональный квадрату собственной частоты $\omega$, и $\mu$ - малый параметр, $\mu^{4}=h^{2} / 12$, где $h$ - толщина оболочки. 
Система (1) рассматривается при одном из 16 типов простых самосопряженных граничных условий [2, с. 81]

$$
\begin{array}{rlrlrl}
u_{1} & =0 & \text { или } & & Q_{1}(u)=0, \\
u_{2}=0 & \text { или } & Q_{2}(u) & =0, \\
u_{3} & =0 & \text { или } & Q_{3}\left(u_{3}\right) & =0, \\
\partial u_{3} / \partial n_{\nu} & =0 & \text { или } & Q_{4}\left(u_{3}\right) & =0 .
\end{array}
$$

Здесь $Q_{i}$ - некоторые дифференциальные выражения (их явный вид можно найти в [2, с. 82-83]), а $n_{\nu}$ - направление внешней нормали к границе $\gamma$ в плоскости, касающейся срединной поверхности в соответствующей точке.

Оператор $L_{\mu}$, порожденный системой (1) и граничными условиями (2)-(5), самосопряжен; он имеет неотрицательные собственные значения

$$
0 \leqslant \lambda_{1} \leqslant \lambda_{2} \leqslant \cdots \leqslant \cdots
$$

с единственной точкой сгущения $\lambda=+\infty$.

Наряду с системой (1), называемой моментной, рассматривается безмоментная (вырожденная) система трех уравнений

$$
L u=\lambda u,
$$

которая получается из (1) при $\mu=0$. Система (7) имеет более низкий порядок и сохраняет самосопряженность при граничных условиях вида

$$
\begin{array}{lll}
u_{1}=0 & \text { или } & Q_{1}(u)=0, \\
u_{2}=0 & \text { или } & Q_{2}(u)=0 .
\end{array}
$$

Соответственно возникает так называемая безмоментная граничная задача (7)-(9), в которую вырождается при $\mu \rightarrow+0$ моментная граничная задача (1), (2)-(5) [2, с. 97-98]. Спектр оператора $L$ уже не является чисто дискретным; подробности см. в $[1,2]$.

Пусть поверхность $S$ выпукла:

$$
R_{1}^{-1}(\alpha, \beta)>0, \quad R_{2}^{-1}(\alpha, \beta)>0, \quad(\alpha, \beta) \in S,
$$

и пусть $\left[\Lambda_{1}, \Lambda_{2}\right]$ - отрезок значений функции

$$
\Omega(\alpha, \beta, \varphi)=\left(1-\sigma^{2}\right)\left(\frac{\cos ^{2} \varphi}{R_{1}(\alpha, \beta)}+\frac{\sin ^{2} \varphi}{R_{2}(\alpha, \beta)}\right)^{2}, \quad(\alpha, \beta) \in S, \varphi \in[0,2 \pi] .
$$

Здесь $R_{i}^{-1}$ - главные кривизны срединной поверхности $S, \sigma$ - коэффициент Пуассона. При любом малом фиксированном $\varepsilon>0$ оператор $L$ в области

$$
0<\lambda<\Lambda_{1}-\varepsilon
$$

имеет конечное число $m$ собственных значений

$$
0 \leqslant \lambda_{1}^{(0)} \leqslant \lambda_{2}^{(0)} \leqslant \cdots \leqslant \lambda_{m}^{(0)}<\Lambda_{1}-\varepsilon .
$$

Соответствующие собственные функции $u_{j}^{(0)}(\alpha, \beta)=\left(u_{1 j}^{(0)}, u_{2 j}^{(0)}, u_{3 j}^{(0)}\right)$ предполагаются нормированными:

$$
\left\|u_{j}\right\|^{2} \equiv \int_{S}\left(u_{1 j}^{2}+u_{2 j}^{2}+u_{3 j}^{2}\right) d s=1
$$


Вырождение при $\mu \rightarrow+0$ задачи (1), (2)-(5) в безмоментную задачу (7)-(9) в области (11) регулярно в смысле Вишика-Люстерника [5]. Отсюда следует, что в области (11) оператор $L_{\mu}$ также имеет $m$ собственных значений $\lambda_{j}^{(\mu)}$, для которых выполняются асимптотические формулы

$$
\lambda_{j}^{(\mu)}=\lambda_{j}^{(0)}+\mu \lambda_{j}^{(1)}+O\left(\mu^{2}\right) .
$$

Справедлива

Теорема 1. При сделанных выше предположениях для первой поправки $\lambda_{j}^{(1)}$ имеет место формула

$$
\lambda_{j}^{(1)}=\sqrt{2} \tau \int_{\gamma}\left(\Omega(\alpha, \beta, \chi)-\lambda_{j}^{(0)}\right)^{3 / 4} u_{3 j}^{(0)^{2}} d \gamma .
$$

Здесь $\chi$ - угол между касательным вектором к координатной линии $\beta=$ const и внешней нормалью $n_{\nu}$. Далее, $\tau=1$, если граничные условия (4), (5) имеют вид $u_{3}=\partial u_{3} / \partial n_{\nu}=0, \tau=0$, если $Q_{3}\left(u_{3}\right)=Q_{4}\left(u_{3}\right)=0$, и $\tau=1 / 2$ в остальных случаях.

Доказательство теоремы потребовало модификации итерационного процесса, предложенного в [5], так как в рассматриваемой задаче малый параметр $h$ входит в систему (1) неоднородно. При этом первая поправка $\lambda_{j}^{(1)}$ оказывается равной контурному интегралу по границе $\gamma$, под знаком которого стоят все компоненты собственной функции $u_{j}^{(0)}$, а также их производные первого порядка. Приведение указанного интеграла к виду (14), единообразному для всех рассматриваемых типов граничных условий, потребовало преодоления значительных технических трудностей. Интересно в связи с этим заметить, что величина $\Omega-\lambda$, стоящая под знаком интеграла в формуле (14), есть главный символ вырожденного оператора $L-\lambda$ (см. [2]). Это обстоятельство роднит формулу (14) с асимптотической формулой для функции распределения $n_{h}(\lambda)$, в которой под знаком интеграла в главном члене также стоит величина $\lambda-\Omega$ (см. $[1$, с. 18$])$.

Численные расчеты подтверждают эффективность формул (13), (14). За недостатком места эти расчеты здесь не приводятся.

\section{ЛитеРАтУРА}

1. Асланян А. Г., Лидский В. Б. Распределение собственных частот тонкий упругих оболочек. Наука, М., 1974. 2. Гольденвейзер А. Л., Лидский В. Б., Товcmuк П. Е. Свободные колебания тонких упругих оболочек. Наука, М., 1979. 3. Власов В. 3. Общая теория оболочек и ее приложение в технике. Гостехиздат, М.-Л., 1949. 4. Новожилов В. В. Теория тонких оболочек. Судпромгиз, Л., 1962. 5. Вишик М. И., Люстерник Л. А. Регулярное вырождение и пограничный слой для линейных дифференциальных уравнений с малым параметром. УМН, 12, вып. 5 (77), 3-122 (1957).

Московский институт радиотехники, электроники и автоматики 